\title{
$\delta$-Catenin Regulates Spine and Synapse Morphogenesis and Function in Hippocampal Neurons during Development
}

\author{
Jyothi Arikkath, ${ }^{1}$ I-Feng Peng, ${ }^{2}$ Yu Gie Ng, ${ }^{1}$ Inbal Israely, ${ }^{3}$ Xin Liu,,${ }^{3,45}$ Erik M. Ullian, ${ }^{2}$ and Louis F. Reichardt ${ }^{1}$ \\ Departments of ${ }^{1}$ Physiology and ${ }^{2}$ Ophthalmology, Beckman Vision Center, University of California, San Francisco, San Francisco, California 94143 , and \\ Departments of ${ }^{3}$ Molecular and Medical Pharmacology and ${ }^{4}$ Pathology and Laboratory Medicine and ${ }^{5}$ Brain Research Institute, University of California, \\ Los Angeles, Los Angeles, California 90095
}

The maintenance of spine and synapse number during development is critical for neuronal circuit formation and function. Here we show that $\delta$-catenin, a component of the cadherin-catenin cell adhesion complex, regulates spine and synapse morphogenesis during development. Genetic ablation or acute knockdown of $\delta$-catenin leads to increases in spine and synapse density, accompanied by a decrease in tetrodotoxin induced spine plasticity. Our results indicate that $\delta$-catenin may mediate conversion of activity-dependent signals to morphological spine plasticity. The functional role of $\delta$-catenin in regulating spine density does not require binding to cadherins, but does require interactions with PDZ domain-containing proteins. We propose that the perturbations in spine and synaptic structure and function observed after depletion of $\delta$-catenin during development may contribute to functional alterations in neural circuitry, the cognitive deficits observed in mutant mice, and the mental retardation pathology of Cri-du-chat syndrome.

\section{Introduction}

Mature neural circuit formation requires accurate generation and elimination of synapses, but molecular mechanisms that control synapse numbers are not well understood. During development, excessive synapses are generated and eliminated through activity-dependent mechanisms, a process critical for neural circuit refinement (Hua and Smith, 2004). The hippocampus has an important role in episodic memory and has been an important site to examine synaptic structure and plasticity (Neves et al., 2008). The majority of hippocampal excitatory synapses are formed on spines. Spine morphology is an important indicator of its functional role (Bourne and Harris, 2008). Spines are highly dynamic and can change structure in response to various physiological stimuli (Alvarez and Sabatini, 2007). Spine head enlargement is associated with long-term potentiation, while spine shrinkage accompanies long-term depression. Structural plasticity of spines is associated with physiological changes in synaptic efficacy (Yuste and Bonhoeffer, 2001; Bourne and Harris, 2007).

Spine abnormalities are associated with several neurological diseases and mental retardation disorders, including Fragile X syndrome, autism

Received Feb. 18, 2009; revised March 11, 2009; accepted March 23, 2009.

J.A. was supported by the Larry L. Hillblom Foundation, and research in L.F.R.'s laboratory was supported by grants from the National Institutes of Health and the Simons Foundation Autism Research Institute. The experiments, except electrophysiology, were designed by J.A. and L.F.R. and performed by J.A. The electrophysiology was performed by I.-F.P. and E.M.U. Y.G.N. provided technical assistance. The $\delta$-catenin null mice were generated and provided by I.I. and X.L. This manuscript was written by J.A. and L.F.R. and revised to incorporate the comments of all the authors. We thank Dr. Seung-hye Lee and other members of the Reichardt and Liu laboratories for comments on this manuscript.

Correspondence should be addressed to either Jyothi Arikkath or Louis F. Reichardt, Department of Physiology, University of California, San Francisco, 1550 Fourth Street, San Francisco, CA 94143. E-mail: Jyothi.arikkath@ucsf.edu or louis.reichardt@ucsf.edu.

DOI:10.1523/JNEUROSCI.0835-09.2009

Copyright $\odot 2009$ Society for Neuroscience $\quad$ 0270-6474/09/295435-08\$15.00/0 spectrum disorders, and neurodegenerative disorders (Pfeiffer and $\mathrm{Hu}-$ ber, 2007; Dindot et al., 2008; Knobloch and Mansuy, 2008).

$\delta$-Catenin, a component of cadherin catenin-cell adhesion complex (Zhou et al., 1997), is one of the genes lost in deletions of chromosome $5 \mathrm{p}$ associated with the Cri-du-chat syndrome (Cerruti Mainardi, 2006). The contribution of $\delta$-catenin to pathology of the disease remains unclear, although there is a strong correlation between loss of $\delta$-catenin and mental retardation (Medina et al., 2000). In addition, a rare copy number variation that disrupts the gene encoding $\delta$-catenin has been implicated in schizophrenia (Vrijenhoek et al., 2008).

$\delta$-Catenin has been localized to dendrites (Arikkath et al., 2008) and synapses (Laura et al., 2002; Silverman et al., 2007). Adult mice that lack $\delta$-catenin have normal basal synaptic transmission at the CA3-CA1 synapse in the hippocampus, but reduced short-term synaptic potentiation and, depending on stimulation protocol, either enhanced or reduced long-term potentiation (Israely et al., 2004). These mice also have severe impairments in cognitive function. Electron microscopic analysis of hippocampal synapses revealed no obvious structural deficits, but there are significant reductions in PSD95 and N-cadherin levels.

Despite these alterations, it is not clear how loss of $\delta$-catenin results in the observed perturbations of synaptic and cognitive function. We hence decided to examine the synaptic role of $\delta$-catenin in more detail. Using mutant mice and acute shRNAmediated knockdown of $\delta$-catenin we show that loss of $\delta$-catenin leads to deficits in hippocampal spine architecture and function. Depletion of $\delta$-catenin leads to increase in spine and synapse density and excitatory synaptic function. Furthermore, loss of $\delta$-catenin inhibits tetrodotoxin-induced spine plasticity. Interestingly, interactions of $\delta$-catenin with PDZ domain-containing proteins, but not with cadherins, appear to be required for its role in controlling spine density and morphogenesis. 
Our results point to a key role for $\delta$-catenin in regulating synaptic structure, function and plasticity during development and provide vital clues to understanding the molecular bases of cognitive deficits in $\delta$-catenin null mice and humans afflicted by Cri-du-chat syndrome.

\section{Materials and Methods}

Antibodies. Antibodies to erbin have been previously described. The mouse monoclonal anti$\delta$-catenin antibody was purchased from BD Transduction Labs. Anti- $\beta$-tubulin antibody (E7) was obtained from the Hybridoma Bank at the University of Iowa. Antibody to PSD95 was from $A B R$ reagents and vGlut1 was from Millipore Bioscience Research Reagents.

Rat and mouse hippocampal cultures. Rat and mouse hippocampal cultures have been previously described (Arikkath et al., 2008).

DNA constructs. All constructs used in this study have been previously described (Arikkath et al., 2008). The $\delta$-catenin $\delta \mathrm{PDZ}^{\star}$ construct was engineered to be resistant to the shRNA. The $\delta$-catenin $\Delta$-arm construct was a kind gift from Dr. Edward Ziff (New York University, New York, NY) and has been previously described (Silverman et al., 2007).

shRNA for $\delta$-catenin. The sequence for this shRNA has been previously described (Arikkath et al., 2008).

Mouse hippocampal culture and transfection. Mouse hippocampal neurons from postnatal day $0 / 1(\mathrm{P} 0 / \mathrm{P} 1) \delta$-catenin $+/+$ and $\delta$-catenin $-/-$ mice were maintained in culture as previously described. Neurons were transfected using Lipofectamine (Invitrogen) with GFP and examined at days in vitro (DIV) 17.

Lysate preparation and Western blot analysis. Hippocampi were collected from P18 $\delta$-catenin $+/+$ and $\delta$-catenin $-/-$ mice and lysed in RIPA buffer with protease inhibitors. The lysates were clarified by centrifugation and the supernatants were loaded on gels for SDS PAGE and immunoblotted with the indicated antibodies. Intensity of bands on the blots was quantified using ImageJ.

Image analysis and statistical methods. All images were taken on an inverted Zeiss Pascal LSM confocal microscope using a $40 \times$ objective and a digital zoom of $4 \times$. Image analysis was done manually or using NIH ImageJ. Images were minimally processed using Adobe Photoshop if required. For spine analysis, dendrites within $80 \mu \mathrm{m}$ of the cell body were used for analysis. For the rat neurons, the $N$ values indicate the number of neurons from three or more independent cultures. For the mouse cultures, the data were obtained from at least three independent control and mutant animals. Statistical significance was assessed using the Student's $t$ test, using a two-tailed test assuming unequal variances. $p$ values obtained from this test are indicated in the figure legends.

Electrophysiology. Spontaneous minature EPSCs (mEPSCs) were recorded from cultured hippocampal neurons by using whole-cell voltageclamp technique at room temperature. Patch pipettes had resistances of 2.5-4.0 $\mathrm{M} \Omega$ and were pulled from borosilicate capillary (WPI). Pipette solution contained $120 \mathrm{~mm}$ K-gluconate, $10 \mathrm{~mm} \mathrm{KCl,} 10 \mathrm{~mm}$ EGTA and $10 \mathrm{~mm}$ HEPES, buffered at $\mathrm{pH}$ 7.3. Bath solution consisted of $120 \mathrm{~mm}$ $\mathrm{NaCl}, 5 \mathrm{~mm} \mathrm{KCl}, 3 \mathrm{~mm} \mathrm{CaCl}, 2 \mathrm{~mm} \mathrm{MgCl}, 10 \mathrm{~mm}$ HEPES, and $1 \mu \mathrm{M}$ tetrodotoxin (TTX). For mEPSC experiments, bicuculline $(20 \mu \mathrm{M})$ was used to block inhibitory inputs. In contrast, during the isolation of spontaneous miniature IPSCs (mIPSCs), AP-V (50 $\mu \mathrm{M})$ and CNQX $(20 \mu \mathrm{M})$ were added in the bath. In the transfected cultures, fluorescent GFP signal was used to identify transfected cells (shRNA, rescue, or control) from nontransfected wild-type neurons. Recordings and data acquisitions were performed by using a MultiClamp 700B amplifier, a Digitdata 1322A converter, and pClamp v9.2 software (all from Molecular Devices). $\delta$-cat $+/+\quad \delta$-cat -/-

$\delta$-cat $+/+\delta$-cat $-/-$

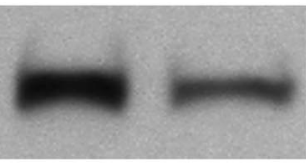

$\alpha \mathrm{N}-$ catenin
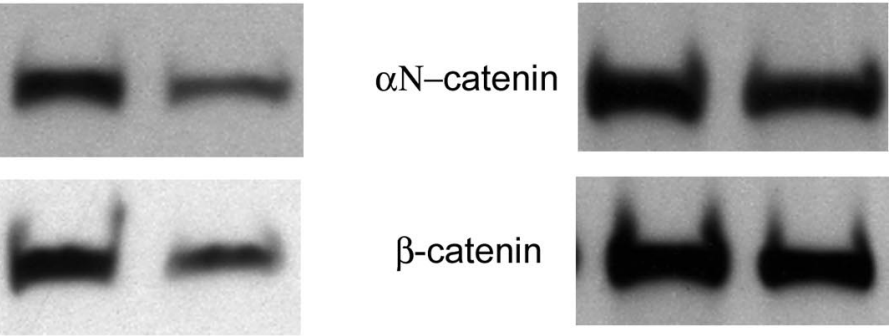

$\beta$-catenin
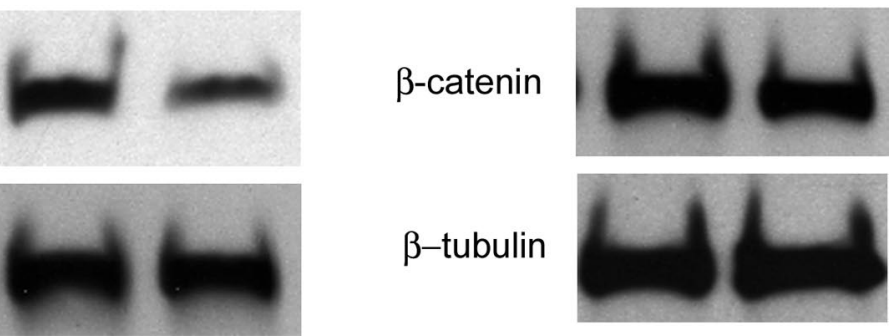

$\beta$-tubulin

Figure 1. Reduction in the levels of $\mathrm{N}$-cadherin and pan-cadherin in hippocampal lysates from $\delta$-catenin $+/+$ and $\delta$-catenin $-/-$ mice. Western blot analysis of hippocampal lysates from P18 $\delta$-catenin $+/+$ and $\delta$-catenin $-/-$ mice with antibodies to $\mathrm{N}$-cadherin, pan-cadherin, p120ctn, $\alpha \mathrm{N}$-catenin, $\beta$-catenin, and $\beta$-tubulin.
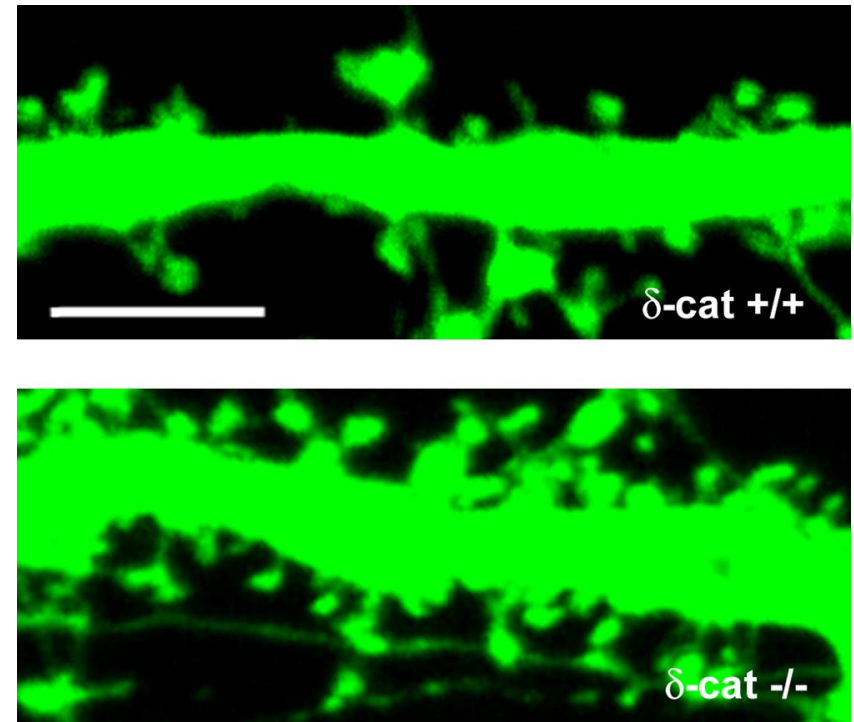

Figure 2. Characteristics of spines from hippocampal neurons from $\delta$-catenin $+/+$ and $\delta$-catenin $-/-$ mice (DIV 17). Representative images of spines from $\delta$-catenin $+/+$ and $\delta$-catenin $-/-$ hippocampal neurons. Scale bar, $5 \mu \mathrm{m}$. $\delta$-cat, $\delta$-Caternin.

\section{Results}

$\delta$-Catenin null mice have reduced levels of cadherin in the hippocampus

Since $\delta$-catenin is a component of the cadherin-catenin cell adhesion complex, we sought to examine the effect of loss of $\delta$-catenin on the components of the cadherin-catenin complex in the hippocampus by Western blot analysis (Fig. 1). To this end, we analyzed hippocampal protein levels of male mice at P18 by Western blot analysis. Immunoblotting analysis $(N=3$ pairs, $\delta$-cat $+/+$ vs $\delta$-cat $-/-$ ) revealed that the levels of cadherin were significantly reduced as examined both with $\mathrm{N}$-cadherin-specific $(100 \pm 3.7 \%$ vs $81.9 \pm 2.4 \%, p<0.05)$ and pan-cadherin $(100 \pm$ $6.4 \%$ vs $72.1 \pm 0.6 \%, p<0.05)$ antibodies. In contrast, there was no significant change in the levels of p120ctn ( $100 \pm 3.4 \%$ vs 93.4 $+/-4.7 \%, p=0.3), \alpha$-catenin $(100 \pm 4.7 \%$ vs $89.2 \pm 3.6 \%, p=$ $0.14)$, and $\beta$-catenin $(100 \pm 0.6 \%$, vs $92.8 \pm 4.7, p=0.26)$. The intensities of the bands on the blots were normalized to the levels of $\beta$-tubulin. Previously, it has been shown that the levels of 

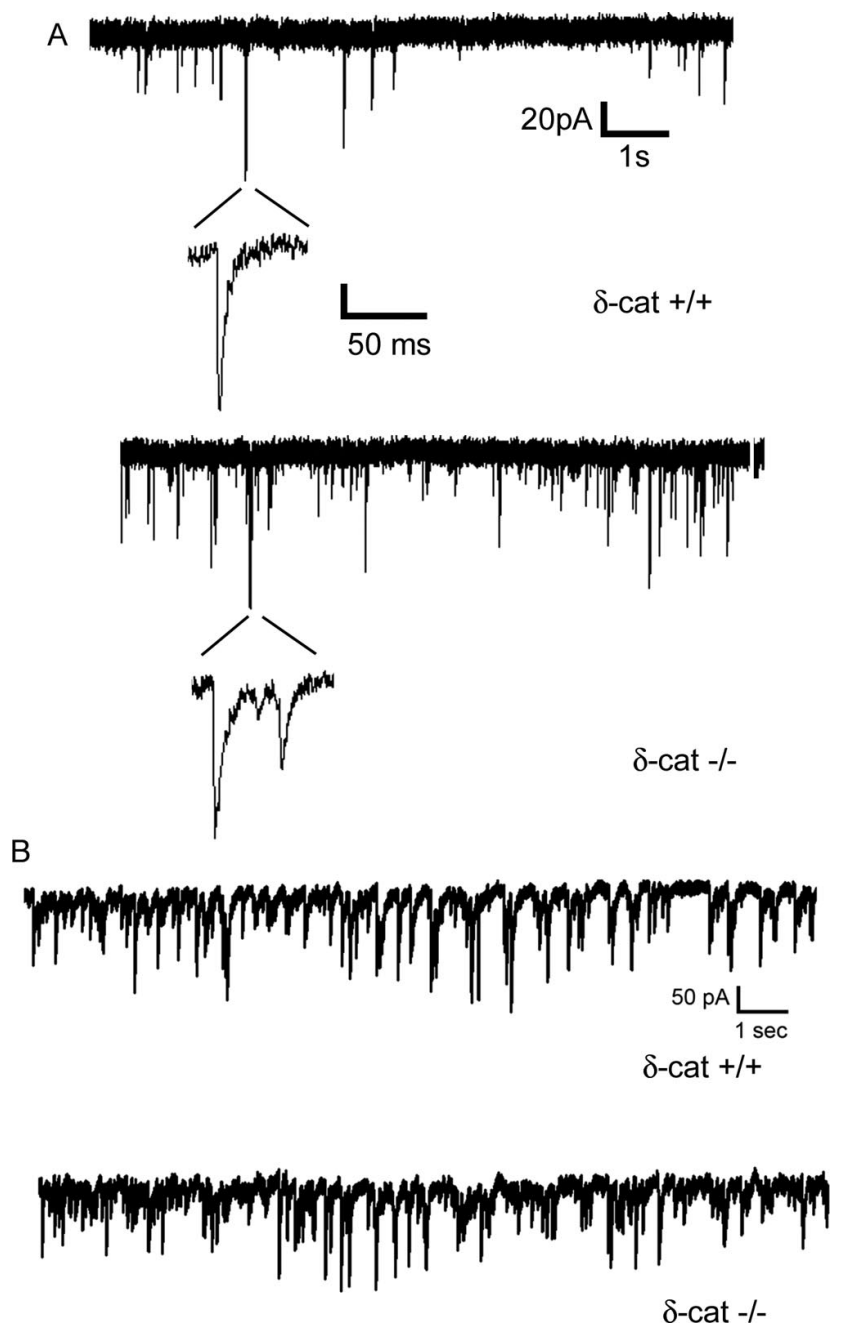

Figure 3. Hippocampal neurons from $\delta$-catenin $-/-$ mice have increased excitatory but not inhibitory synaptic function. $A$, Representative traces of mEPSCs from $\delta$-catenin $+/+$ and $\delta$-catenin $-/-$ neurons. $B$, Representative traces of mIPSCs from $\delta$-catenin $+/+$ and $\delta$-catenin $-/-$ neurons. $\delta$-cat, $\delta$-Catenin.

cadherin are reduced in adult $\delta$-catenin null mice (Israely et al., 2004). Together, these results suggest that the loss of $\delta$-catenin leads to a persistent reduction in the levels of cadherin both during and after development.

Hippocampal neurons from $\boldsymbol{\delta}$-catenin null mice have increased spine density, with decreased spine headwidth and length

To understand the role of $\delta$-catenin in spine and synapse morphogenesis, we examined spines in hippocampal neurons in culture from control and $\delta$-catenin null mice at DIV 17. Cultured mouse hippocampal neurons were transfected with EGFP to visualize neuronal morphology. Close examination indicated that, compared with WT controls, there was an increase in spine density in $\delta$-catenin null neurons (spines/ $10 \mu \mathrm{m}, 5.3 \pm 0.5$ vs $7.2 \pm$ $0.5, p=0.0085)$ with a concomitant decrease in spine headwidth $(0.83 \pm 0.02 \mu \mathrm{m}$, vs $0.72 \pm 0.01 \mu \mathrm{m}, p<0.001)$ and length $(1.67 \pm 0.04 \mu \mathrm{m}$, vs $1.5 \pm 0.03 \mu \mathrm{m}, p<0.001)$ (Fig. 2). These results indicate that loss of $\delta$-catenin leads to an increase in the density of spines and excitatory synapses in hippocampal neurons.
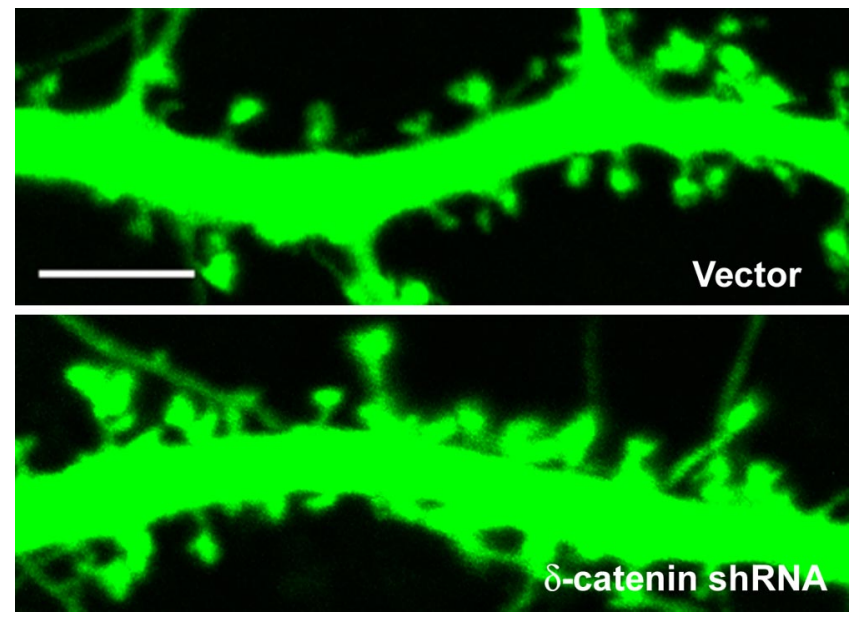

Figure 4. Acute shRNA-mediated knockdown of $\delta$-catenin leads to an increase in spine density. Representative images of spines from neurons transfected with the vector or shRNA to $\delta$-catenin at DIV 11 and examined at DIV 17. Scale bar, $5 \mu \mathrm{m}$.

Increased spine density in $\boldsymbol{\delta}$-catenin null neurons is accompanied by increased excitatory synaptic activity with no changes in the AMPA/NMDA ratio

To determine whether the increase in spine density in cultured hippocampal neurons lacking $\delta$-catenin is accompanied by an increase in synaptic function, we compared the frequency and amplitude of mEPSCs from control and $\delta$-catenin null neurons at DIV 17-18. Consistent with the increase in spine density, we observed in neurons lacking $\delta$-catenin an increase in the frequency of mEPSCs with no significant change in the amplitude (Fig. 3A) [frequency (events/min): $292 \pm 66$ vs $589 \pm 129, p=$ 0.014 ; amplitude $(\mathrm{pA}): 20.29 \pm 1.54$, vs $22.22 \pm 1.46, p=0.369$, $n>20$ neurons]. Furthermore, to examine whether these effects are specific for excitatory synapses, we recorded mIPSCs in neurons from $\delta$-catenin $+/+$ and $-/$ - neurons. Unlike the effects of loss of $\delta$-catenin on mEPSCs, we observed no alterations in the frequency or amplitude of mIPSCs in neurons from $\delta$-catenin $-/-$ neurons (Fig. 3B). [Frequency (events/min): $515 \pm 86.2$, vs $-612 \pm 115, \mathrm{n}>20$ neurons, $p=0.516$; amplitude (pA): $50.7 \pm$ 4.89 , vs $53.1 \pm 2.4, n>20$ neurons, $p=0.641$ ). These results suggest that there is a higher density of excitatory, but not inhibitory synapses in cultures of mutant neurons.

$\delta$-Catenin has previously been shown to enhance surface expression of the AMPA receptor subunits GluR2 and Glur3 via interactions with AMPA receptor binding protein (ABP) and glutamate receptor interacting protein (GRIP) (Silverman et al., 2007). In organotypic cultures, overexpression or shRNAmediated depletion of $\delta$-catenin has been shown to increase or decrease, respectively, the synaptic AMPA, but not NMDA response (Ochiishi et al., 2008). To examine the effects of loss of $\delta$-catenin on the AMPA and NDMA currents, we examined the ratio of AMPA and NMDA currents in dissociated hippocampal neuronal cultures at DIV $17-18$ from control and $\delta$-catenin null mice $[\delta$-catenin $+/+-3.63 \pm 0.59(N=15), \delta$-catenin $-/-$ $-5.20 \pm 1.12(n=17), p=0.242]$. Contrary to our expectations, there was no significant change in the ratio of AMPA to NMDA in these cultures, indicating that loss of $\delta$-catenin at this stage during development does not appreciably alter the surface expression of AMPA receptors. Among other possibilities, the discrepancy between our observations and those of others cited above may reflect compensatory changes in expression of other $\delta$-catenin homologues in the mutant neurons. 
Acute shRNA-mediated knockdown of $\delta$-catenin leads to an increase in spine density with a decrease in spine headwidth and length

To examine whether the increase in spine density in $\delta$-catenin null neurons was a cell autonomous effect and could be observed after acute depletion of $\delta$-catenin, we examined the effect of acute knockdown of $\delta$-catenin on spine parameters in cultured rat hippocampal neurons. To this end, rat hippocampal neurons were transfected with shRNA to $\delta$-catenin at DIV 11 and examined at DIV 17 (Fig. 4). Consistent with the phenotype in neurons from $\delta$-catenin null mice, neurons that express the shRNA to $\delta$-catenin exhibited increased spine density (spines/10 $\mu \mathrm{m}$ : vector, $5.5 \pm 0.3$; shRNA, $7.3 \pm 0.5 ; p=$ $0.005)$ with concomitant decreases in spine headwidth (vector, $0.82 \pm 0.01 \mu \mathrm{m}$; shRNA, $0.69 \pm 0.01 \mu \mathrm{m}, p<0.001)$ and length (vector, $1.62 \pm 0.02$; shRNA, $1.53 \pm 0.03 ; p<0.02)$. The decrease in spine length was significant, but it was not as striking as in the neurons from $\delta$-catenin null mice. These results suggest that both acute knockdown and complete loss of $\delta$-catenin lead to similar alterations in spine morphology. Furthermore, as only a small percentage of neurons are transfected in these cultures, the functional role of $\delta$-catenin in spine morphogenesis appears to be cell autonomous. Since an increase in spine density was the most dramatic morphological phenotype, we focused further analysis on this parameter.

\section{Acute shRNA-mediated knockdown of} $\delta$-catenin is accompanied by an increase in excitatory synaptic density and function

To examine the effects of acute knockdown of $\delta$-catenin on excitatory synapse formation, we examined the effect of $\delta$-catenin depletion on the density of an excitatory postsynaptic marker PSD95 in neurons expressing the shRNA to $\delta$-catenin (Fig. $5 A$ ). Given the low transfection efficiency of the shRNA in these neurons, we expected the results to reflect cell autonomous effects of $\delta$-catenin depletion. Consistent with an increase in spine density, acute knockdown of $\delta$-catenin was accompanied by an increase in the density of PSD95 puncta (puncta/10 $\mu \mathrm{m}$ : vector, $4.42 \pm$ 0.33 ; shRNA, $9.92 \pm 0.8 ; p<0.0001)$. To examine whether these PSD95 puncta represented synapses, we examined the density of the presynaptic excitatory marker, vGlut 1 in neurons expressing the shRNA to $\delta$-catenin (Fig. $5 B$ ). These studies showed that the dendrites of neurons expressing shRNA to $\delta$-catenin also were contacted by an increased density of presynaptic excitatory puncta (puncta/10 $\mu \mathrm{m}$ : vector, $3.8 \pm 0.4$; shRNA, $6.5 \pm 0.5 ; p<0.0005)$, suggesting that the increase in spine density that arises from acute knockdown of $\delta$-catenin is ac- companied by an increase in synaptic density. To obtain more definitive evidence, we recorded mEPSCs from neurons expressing vector, shRNA to $\delta$-catenin or shRNA to $\delta$-catenin and a full-length shRNA-resistant construct of $\delta$-catenin. Acute knockdown of $\delta$-catenin leads to an increase in the frequency of the mEPSCs with no significant change in mEPSC average amplitude (Fig. 5C-E). This increase in mEPSC frequency was abolished in neurons by expression of an shRNA-resistant construct of $\delta$-catenin, confirming the specificity of the shRNA-mediated knockdown [frequency (events/min): vector, $327 \pm 65$; shRNA, $566 \pm 74$; shRNA + $\delta$-catenin ${ }^{\star}, 252 \pm 29$; amplitude $(\mathrm{pA})$ : vector, $18.9 \pm 1.3$; shRNA, $21.0 \pm 1.0 ;$ shRNA $+\delta$-catenin ${ }^{*}, 18.9 \pm 1.4, n=5-8$ neurons; $p$ values: vector vs shRNA, 0.025 ; shRNA vs shRNA plus $\delta$-catenin, 0.017 ; vector vs shRNA plus $\delta$-catenin $\left.{ }^{\star}, 0.05\right]$. 
A

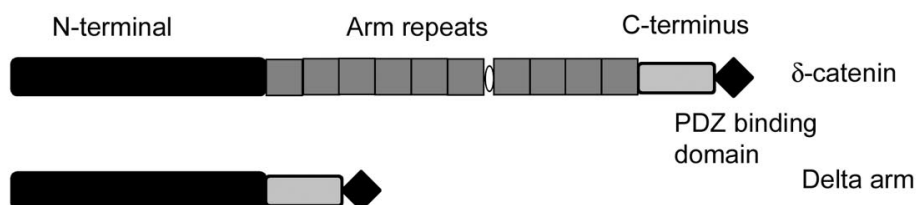

B
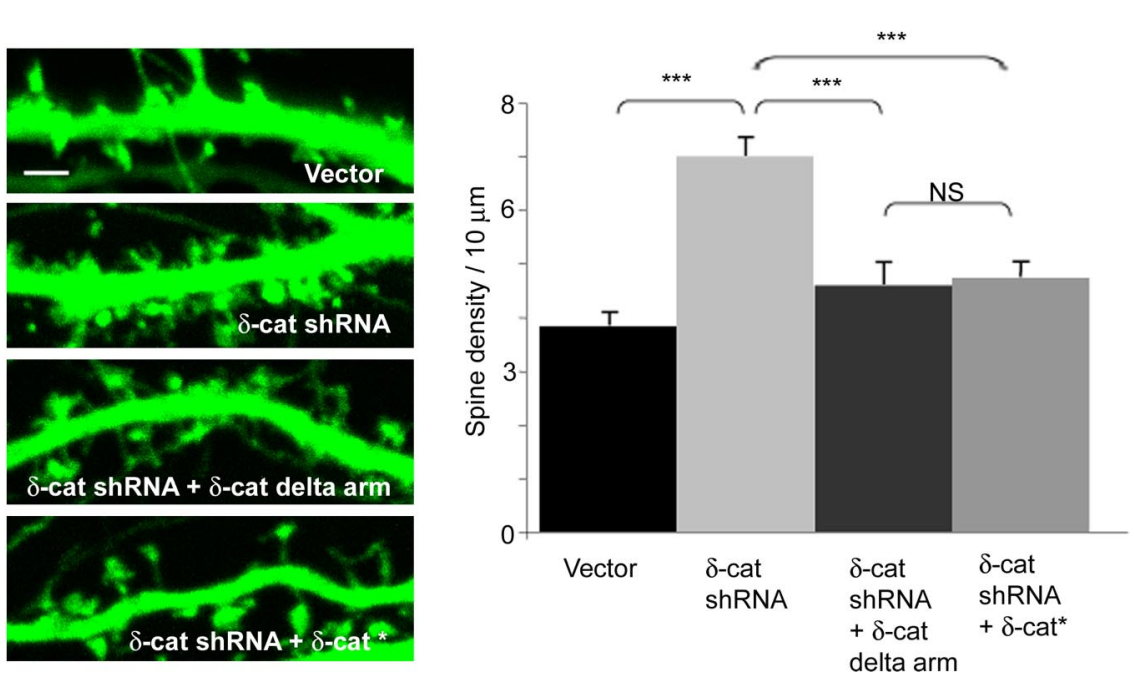

Figure 6. Acute $\delta$-catenin knockdown-mediated increase in spine density is independent of binding of cadherin. $\boldsymbol{A}$, Schematic of constructs of full-length $\delta$-catenin and $\Delta$-arm $\delta$-catenin. $\boldsymbol{B}$, Representative images of spines from rat hippocampal neurons transfected with vector, $\delta$-catenin shRNA, $\delta$-catenin shRNA plus $\delta$-catenin $\Delta$-arm, or $\delta$-catenin shRNA plus $\delta$-catenin* at DIV 11 and examined at DIV 17. Scale bar, $2 \mu \mathrm{m}$. C, Quantitation of spine density (per $10 \mu \mathrm{m}$ ) from rat hippocampal neurons expressing vector, shRNA, shRNA plus $\delta$-catenin $\Delta$-arm, or shRNA plus $\delta$-catenin* $\left.{ }^{* * *} p<0.006\right)$. $\delta$-cat, $\delta$-Catenin.

Together, these results confirm specificity of the shRNAmediated knockdown and demonstrate that acute knockdown of $\delta$-catenin leads to an increase in spine and synapse density and function, similar to that observed in neurons from mice that lack $\delta$-catenin. Furthermore, these results also confirm that these effects of loss of $\delta$-catenin are cell autonomous.

\section{Regulation of spine density by $\delta$-catenin does not require cadherin binding}

Since $\delta$-catenin controls stability of the surface cadherin complex (Davis et al., 2003), we sought to determine whether the increase in spine density that accompanies loss of $\delta$-catenin requires cadherin binding. To this end, we compared the abilities of shRNAresistant $\delta$-catenin and shRNA-resistant $\delta$-catenin lacking the cadherin interaction site $(\Delta$-ARM) to suppress the increase in spine density observed after shRNA-dependent $\delta$-catenin depletion (Fig. $6 A, B$ ). Similar to the results in Figure 3, knockdown of $\delta$-catenin resulted in an increase in spine density. This increase in spine density was prevented by coexpression of shRNA-resistant full-length $\delta$-catenin or a $\delta$-catenin lacking the ARM repeats (spines/10 $\mu \mathrm{m}$ : vector, $3.7 \pm 0.28$; shRNA, $6.9 \pm 0.34$; shRNA plus $\delta$-catenin $\Delta$-arm, $5.27 \pm 0.43$; shRNA plus full-length $\delta$-catenin ${ }^{*}, 4.82 \pm 0.33 ; p<0.006$ for shRNA vs shRNA plus $\delta$-catenin $\Delta$-arm, $p=0.4$ for shRNA plus $\delta$-catenin $\Delta$-arm vs shRNA plus $\delta$-catenin ${ }^{\star} ; N=15$ neurons). These results suggest that $\delta$-catenin controls hippocampal spine density independent of cadherin binding although it is possible that cadherin binding increases the efficiency with which endogenous $\delta$-catenin regulates spine density. Furthermore, these results confirm the specificity of the shRNA-mediated knockdown.

\section{$\delta$-Catenin regulates spine density through PDZ motif interactions with PDZ containing proteins}

To further examine mechanisms through which $\delta$-catenin regulates spine density, we examined the abilities of a deletion series of shRNA-resistant $\delta$-catenin constructs to restore normal spine density in shRNA-depleted hippocampal neurons (Fig. $7 A, B$ ). As expected, shRNAmediated $\delta$-catenin depletion significantly increased spine density and this was prevented by coexpression of shRNAresistant $\delta$-catenin (spine density/10 $\mu \mathrm{m}$ : vector, $5.24 \pm 0.34$; shRNA, $7.55 \pm 0.44$; shRNA plus $\delta$-catenin, $4.57 \pm 0.27$; shRNA vs shRNA plus $\delta$-catenin ${ }^{*}, p<$ $0.0001)$. Results demonstrate that deletion of the C-terminal 4 aa alone ( $\triangle \mathrm{PDZ}$ ) is sufficient to prevent an shRNA-resistant $\delta$-catenin construct from restoring normal spine density (shRNA plus $\mathrm{N}$ terminus, $6.76 \pm 0.47 ;$ shRNA plus N-Arm, $7.72 \pm$ 0.44 ; shRNA plus $\delta$-catenin $\Delta$ PDZ, $6.81 \pm$ $0.32 ; p>0.05$ vs shRNA). Longer C-terminal deletions also failed to restore spine density. None of the deletion constructs, including that lacking the C-terminal 4 aa significantly suppressed spine density in neurons depleted of endogenous $\delta$-catenin. As the C-terminal 4 aa are known to mediate interactions with several PDZ-domain containing proteins, including AMPA receptor-binding protein, glutamate receptor-interacting protein, densin, and erbin (Silverman et al., 2007; Arikkath et al., 2008 ), these data strongly suggest that $\delta$-catenin controls spine density through interactions with a PDZ-domain-containing protein.

\section{Acute knockdown of $\delta$-catenin inhibits TTX-mediated morphological plasticity}

Dendritic spines are not merely static structures but can be structurally modified by various physiological stimuli, including synaptic activity (Segal, 2005). Inhibition of activity through TTX application has been previously shown to convert many hippocampal neuron spines into filopodial-like structures and reduce the amount of $\alpha \mathrm{N}$-catenin present in these structures (Papa and Segal, 1996; Abe et al., 2004). Overexpression of $\alpha \mathrm{N}$-catenin suppresses this change in spine shape. Activation of NMDA receptors has been shown to recruit $\beta$-catenin into spine heads, promote spine head enlargement, and stabilize synaptic $\mathrm{N}$-cadherin through regulation of $\beta$-catenin phosphorylation, while absence of $\beta$-catenin increases the proportion of filopodiallike spines and prevents regulation of synaptic strength in response to manipulation of synaptic activity (Okuda et al., 2007; 
Tai et al., 2007). To determine whether $\delta$-catenin, previously shown to control surface cadherin levels (Davis et al., 2003), regulates spine plasticity, we examined the effect of silencing neuronal activity by TTX in control and $\delta$-catenin depleted neurons. To this end, we transfected rat hippocampal neurons at DIV 11 with vector or shRNA and treated them with TTX from DIV 18-21 after which neurons were fixed and processed for imaging. In control vector transfected neurons, we observed an increase in the spine length after TTX treatment $(1.55 \pm 0.035 \mu \mathrm{m}$, to $1.99 \pm 0.066, p<0.0001)$. However, shRNA-mediated $\delta$-catenin depletion prevented the increase in spine length after TTX application observed in controls $(1.53 \pm 0.03 \mu \mathrm{m}$, TTX $-1.54 \pm 0.04, p=$ $0.9)$ (Fig. 8). These results indicate that the spines of $\delta$-catenin-depleted neurons are resistant to activity-induced structural changes. This supports the idea that $\delta$-catenin may function as a regulator to convert activity-dependent signals into alterations in spine morphology. As cadherins have been implicated as mediators of activity-driven changes in spines, it will be interesting to determine which domains of $\delta$-catenin are required to regulate activity-induced structural changes in the spine and whether $\delta$-catenin functions in the same pathway controlled by $\mathrm{N}$-cadherin (Okamura et al., 2004)

\section{Discussion}

In this study, we show that loss of $\delta$-catenin throughout development in a knock-out mouse leads to increases in spine and synapse density in vivo and in vitro. Acute shRNA knockdown in vitro results in the same increases. $\delta$-catenin knockdown also inhibits changes in spine morphology as a result of activity silencing.

Our results show that interactions mediated through the C-terminal 4 aa PDZ binding motif of $\delta$-catenin are required for $\delta$-catenin-mediated regulation of spine density. This suggests strongly that interactions with PDZ domain proteins are required for this function. $\delta$-catenin is known to bind to several PDZ containing proteins including erbin, densin-180, S-SCAM, PAPIN, ABP, and GRIP (Ide et al., 1999; Deguchi et al., 2000; Izawa et al., 2002; Laura et al., 2002). It is not certain, however, which, if any of these proteins control this activity of $\delta$-catenin, In contrast, the data suggest that $\delta$-catenin association with cadherins is not required since a deletion construct lacking the cadherin interaction domain appears able to regulate spine density normally. It is becoming increasingly clear that although the catenins are associated with the cadherins, the catenins can have functional roles that are independent of their ability to bind cadherin and regulate cell adhesion (Arikkath and Reichardt, 2008; Kim et al., 2008).

Our results are in contrast with previously published results that show that siRNA-mediated knockdown of $\delta$-catenin leads to a decrease in the spine/protrusion density in neurons at DIV 20
(Abu-Elneel et al., 2008). The cause of this discrepancy is not certain, but our data in vitro using neurons from the $\delta$-catenin mutant or neurons acutely depleted of $\delta$-catenin are selfconsistent. Differences in culture conditions or time of analysis may explain this discrepancy.

Similarly, our electrophysiological analyses of synaptic properties of neurons from the $\delta$-catenin mutant appears inconsistent with previous observations indicating that $\delta$-catenin promotes surface expression of AMPA receptors and results in enhanced AMPA receptor-mediated synaptic currents (Silverman et al., 2007). We observed no differences in the AMPA/NMDA current ratio in neurons from $\delta$-catenin null mice. Among many possibilities, expression of one or more $\delta$-catenin homolog, such as ARVCF or p0071, may be elevated in the $\delta$-catenin mutant, resulting in compensation not observed in cultures in which $\delta$-catenin is depleted through shRNA treatment. Since it has been previously shown that $\delta$-catenin-mediated trafficking of AMPA receptors is mediated through the PDZ-domain containing AMPA receptorbinding proteins ABP and GRIP, it is unlikely that compensation occurs through p120ctn which lacks a PDZ domain interaction motif. In addition, we did not observe a significant change in p120ctn levels in hippocampal lysates from 
A
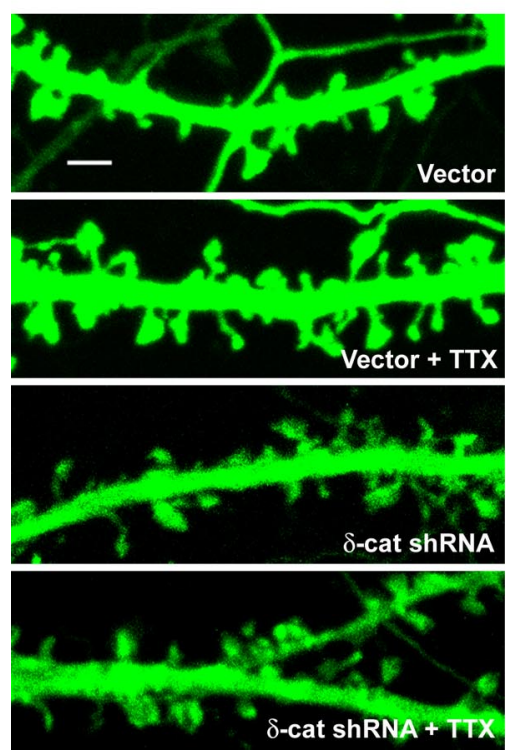

$\delta$-catenin inhibits tetrodotoxin induced spine plasticity. This suggests that $\delta$-catenin may play an important role in the transduction of stimuli to morphological changes in spine structure. This is an important function for physiological plasticity and absence of $\delta$-catenin may contribute to impairments in the formation and function of neural circuitry during development. As cadherins have been implicated as mediators of activity-driven changes in spines, it will be interesting to determine which domains of $\delta$-catenin are required to regulate activity-induced structural changes in the spine and whether $\delta$-catenin functions in the same pathway controlled by N-cadherin (Okamura et al., 2004).

The loss of activity-dependent plasticity of spines in the absence of $\delta$-catenin suggests that loss of $\delta$-catenin promotes spine stability. It is likely that the excessive spines that are formed in the absence of $\delta$-catenin have increased stability and $\delta$-catenin may have a role in synapse elimination during development in the hippocampus. Interestingly enough, schizo-

$\delta$-catenin null mice (Fig. 1). Unfortunately, antibodies specific for ARVCF and p0071 have not been described.

Our results are in striking contrast to previously published data on the p120ctn, a structural homolog of $\delta$-catenin, in spine and synapse formation (Elia et al., 2006). Loss of p120ctn results in significant reduction in spine and synapse densities in vivo and in vitro. In vitro analyses indicate that p120ctn controls spine density through regulation of active Rho levels. Consistent with this, loss of p120ctn leads to a global increase in the levels of active Rho, with a concomitant decrease in the levels of active Rac (Elia et al., 2006). However, we have previously shown (Arikkath et al., 2008) that there are no obvious alterations in the levels of active Rac or Rho in neurons from $\delta$-catenin null mice at the stage where the increases in spine density are observed. Unlike p120ctn, $\delta$-catenin contains a C-terminal PDZ domain interaction motif and is capable of binding a large number of PDZ domain-containing proteins, some of which, such as erbin, clearly do not associate with p120ctn (Laura et al., 2002). Together, these results point to strikingly different mechanisms through which two members of the same family of proteins, p120ctn and $\delta$-catenin, regulate spine morphogenesis. Loss of these proteins has opposing effects on spine and synapse density. They appear to regulate spine and synapse morphogenesis in hippocampal neurons by at least partially different mechanisms.

Our observations confirm previous data demonstrating that, similar to p120ctn deletion (Elia et al., 2006), the absence of $\delta$-catenin reduces the level of $\mathrm{N}$-cadherin (Israely et al., 2004). It has been previously shown that p120ctn family members, including $\delta$-catenin and $\mathrm{p} 120 \mathrm{ctn}$, reduce endocytosis of surface cadherins (Davis et al., 2003; Xiao et al., 2003). It is thus likely that loss of $\delta$-catenin reduces cadherin stability, thereby decreasing steady state levels. How this reduction in cadherin stability influences synaptic function remains to be examined.

Spines are highly plastic and show morphological and functional alterations in response to physiological insults and stimuli (Engert and Bonhoeffer, 1999). Our data show that loss of phrenia has been suggested to be attributed to deficits in synapse pruning (Keshavan et al., 1994; Hoffman and McGlashan, 1997) and a number of mental retardations have been associated with aberrant spine structure and function.

It has previously been shown that mice that lack $\delta$-catenin have severe cognitive abnormalities (Israely et al., 2004). However, the molecular bases of these deficits have been unclear. It is widely known that spine abnormalities are common in a number of genetic mental retardation syndromes. We propose that the abnormal spine morphology, function and plasticity that occurs with loss of $\delta$-catenin during synaptogenesis contributes to deficits in circuit formation and function and contribute to the cognitive abnormalities observed in adult mice that lack $\delta$-catenin. Furthermore, we propose that, since the $\delta$-catenin gene is one of several deleted in Cri-du-chat patients, spine and synapse abnormalities resulting from absence of this gene contribute to the mental retardation pathology of this devastating disorder.

\section{References}

Abe K, Chisaka O, Van Roy F, Takeichi M (2004) Stability of dendritic spines and synaptic contacts is controlled by alpha N-catenin. Nat Neurosci 7:357-363.

Abu-Elneel K, Ochiishi T, Medina M, Remedi M, Gastaldi L, Caceres A, Kosik KS (2008) A delta-catenin signaling pathway leading to dendritic protrusions. J Biol Chem 283:32781-32791.

Alvarez VA, Sabatini BL (2007) Anatomical and physiological plasticity of dendritic spines. Annu Rev Neurosci 30:79-97.

Arikkath J, Reichardt LF (2008) Cadherins and catenins at synapses: roles in synaptogenesis and synaptic plasticity. Trends Neurosci 31:487-494.

Arikkath J, Israely I, Tao Y, Mei L, Liu X, Reichardt LF (2008) Erbin controls dendritic morphogenesis by regulating localization of delta-catenin. J Neurosci 28:7047-7056.

Bourne J, Harris KM (2007) Do thin spines learn to be mushroom spines that remember? Curr Opin Neurobiol 17:381-386.

Bourne JN, Harris KM (2008) Balancing structure and function at hippocampal dendritic spines. Annu Rev Neurosci 31:47-67.

Cerruti Mainardi P (2006) Cri du Chat syndrome. Orphanet J Rare Dis 1:33.

Davis MA, Ireton RC, Reynolds AB (2003) A core function for p120-catenin in cadherin turnover. J Cell Biol 163:525-534. 
Deguchi M, Iizuka T, Hata Y, Nishimura W, Hirao K, Yao I, Kawabe H, Takai Y (2000) PAPIN. A novel multiple PSD-95/Dlg-A/ZO-1 protein interacting with neural plakophilin-related armadillo repeat protein/deltacatenin and p0071. J Biol Chem 275:29875-29880.

Dindot SV, Antalffy BA, Bhattacharjee MB, Beaudet AL (2008) The Angelman syndrome ubiquitin ligase localizes to the synapse and nucleus, and maternal deficiency results in abnormal dendritic spine morphology. Hum Mol Genet 17:111-118.

Elia LP, Yamamoto M, Zang K, Reichardt LF (2006) p120 catenin regulates dendritic spine and synapse development through Rho-family GTPases and cadherins. Neuron 51:43-56.

Engert F, Bonhoeffer T (1999) Dendritic spine changes associated with hippocampal long-term synaptic plasticity. Nature 399:66-70.

Hoffman RE, McGlashan TH (1997) Synaptic elimination, neurodevelopment, and the mechanism of hallucinated "voices" in schizophrenia. Am J Psychiatry 154:1683-1689.

Hua JY, Smith SJ (2004) Neural activity and the dynamics of central nervous system development. Nat Neurosci 7:327-332.

Ide N, Hata Y, Deguchi M, Hirao K, Yao I, Takai Y (1999) Interaction of S-SCAM with neural plakophilin-related Armadillo-repeat protein/deltacatenin. Biochem Biophys Res Commun 256:456-461.

Israely I, Costa RM, Xie CW, Silva AJ, Kosik KS, Liu X (2004) Deletion of the neuron-specific protein delta-catenin leads to severe cognitive and synaptic dysfunction. Curr Biol 14:1657-1663.

Izawa I, Nishizawa M, Ohtakara K, Inagaki M (2002) Densin-180 interacts with delta-catenin/neural plakophilin-related armadillo repeat protein at synapses. J Biol Chem 277:5345-5350.

Keshavan MS, Anderson S, Pettegrew JW (1994) Is schizophrenia due to excessive synaptic pruning in the prefrontal cortex? The Feinberg hypothesis revisited. J Psychiatr Res 28:239-265.

Kim H, Oh M, Lu Q, Kim K (2008) E-Cadherin negatively modulates deltacatenin-induced morphological changes and RhoA activity reduction by competing with p190RhoGEF for delta-catenin. Biochem Biophys Res Commun 377:636-641.

Knobloch M, Mansuy IM (2008) Dendritic spine loss and synaptic alterations in Alzheimer's disease. Mol Neurobiol 37:73-82.

Laura RP, Witt AS, Held HA, Gerstner R, Deshayes K, Koehler MF, Kosik KS, Sidhu SS, Lasky LA (2002) The Erbin PDZ domain binds with high affinity and specificity to the carboxyl termini of delta-catenin and ARVCF. J Biol Chem 277:12906-12914.

Medina M, Marinescu RC, Overhauser J, Kosik KS (2000) Hemizygosity of delta-catenin (CTNND2) is associated with severe mental retardation in cri-du-chat syndrome. Genomics 63:157-164.

Neves G, Cooke SF, Bliss TV (2008) Synaptic plasticity, memory and the hippocampus: a neural network approach to causality. Nat Rev Neurosci 9:65-75.

Ochiishi T, Futai K, Okamoto K, Kameyama K, Kosik KS (2008) Regulation of AMPA receptor trafficking by delta-catenin. Mol Cell Neurosci 39:499-507.

Okamura K, Tanaka H, Yagita Y, Saeki Y, Taguchi A, Hiraoka Y, Zeng LH, Colman DR, Miki N (2004) Cadherin activity is required for activityinduced spine remodeling. J Cell Biol 167:961-972.

Okuda T, Yu LM, Cingolani LA, Kemler R, Goda Y (2007) beta-Catenin regulates excitatory postsynaptic strength at hippocampal synapses. Proc Natl Acad Sci U S A 104:13479-13484.

Papa M, Segal M (1996) Morphological plasticity in dendritic spines of cultured hippocampal neurons. Neuroscience 71:1005-1011.

Pfeiffer BE, Huber KM (2007) Fragile X mental retardation protein induces synapse loss through acute postsynaptic translational regulation. J Neurosci 27:3120-3130.

Segal M (2005) Dendritic spines and long-term plasticity. Nat Rev Neurosci 6:277-284.

Silverman JB, Restituito S, Lu W, Lee-Edwards L, Khatri L, Ziff EB (2007) Synaptic anchorage of AMPA receptors by cadherins through neural plakophilin-related arm protein AMPA receptor-binding protein complexes. J Neurosci 27:8505-8516.

Tai CY, Mysore SP, Chiu C, Schuman EM (2007) Activity-regulated N-cadherin endocytosis. Neuron 54:771-785.

Vrijenhoek T, Buizer-Voskamp JE, van der Stelt I, Strengman E, Sabatti C, Geurts van Kessel A, Brunner HG, Ophoff RA, Veltman JA (2008) Recurrent CNVs disrupt three candidate genes in schizophrenia patients. Am J Hum Genet 83:504-510.

Xiao K, Allison DF, Buckley KM, Kottke MD, Vincent PA, Faundez V, Kowalczyk AP (2003) Cellular levels of p120 catenin function as a set poin for cadherin expression levels in microvascular endothelial cells. J Cell Biol 163:535-545.

Yuste R, Bonhoeffer T (2001) Morphological changes in dendritic spines associated with long-term synaptic plasticity. Annu Rev Neurosci 24:1071-1089.

Zhou J, Liyanage U, Medina M, Ho C, Simmons AD, Lovett M, Kosik KS (1997) Presenilin 1 interaction in the brain with a novel member of the Armadillo family. Neuroreport 8:2085-2090. 